\title{
Prediction of Physical-Chemical and Fire Hazard Characteristics by Carbon Chain Rules. 1. Alkanals
}

\author{
Kirill S. Alexeev ${ }^{\mathrm{a}, \mathrm{b}}$, \\ Sergey G. Alexeev ${ }^{\text {*a,c }}$ and Nicolay M. Barbin ${ }^{\mathrm{b}, \mathrm{c}}$ \\ ${ }^{a}$ Science and Engineering Centre \\ "Reliability and Safety of Large Systems" UB RAS \\ 54a Studencheskaya Str., Yekaterinburg, 620049, Russia \\ ${ }^{b}$ Ural State Agrarian University \\ 42 Karla Libknekhta Str., Yekaterinburg, 620075, Russia \\ ${ }^{c}$ Ural Institute of State Fire Service of EMERCOM of Russia \\ 22 Mira Str., Yekaterinburg, 620062 Russia
}

Received 12.03.2018, received in revised form 18.04.2018, accepted 31.05.2018

\begin{abstract}
Investigations of the dependence of physico-chemical and fire hazard properties from the chemical structure of alkanals are carried out. Forecasting the boiling temperature, the flash point, the temperature and concentration flammability limits, the heats of combustion and vaporization is performed by the rules of the carbon chain. For the convenience of practical application of the rules of the carbon chain, the following empirical equations for the calculation of physico-chemical and fire hazard indices from the conventional carbon chain and from the number of carbon atoms are proposed. A comparative analysis of the proposed methods for calculating the flash point and the methods of GOST 12.1.044-89, Mendeleev and ACD/Lab 2014 is carried out. It is shown that the new methods basically give more accurate calculation results than the comparison design procedures.
\end{abstract}

Keywords: boiling point, flash point, temperature flammability limits, lammability limits, heat of combustion, heat of vaporization, aldehyde.

Citation: Alexeev K.S., Alexeev S.G., Barbin N.M. Prediction of physical-chemical and fire hazard characteristics by carbon chain rules. 1. Alkanals, J. Sib. Fed. Univ. Chem., 2018, 11(2), 219-229. DOI: 10.17516/1998-2836-0070.

(C) Siberian Federal University. All rights reserved

* Corresponding author E-mail address: 3608113@mail.ru 


\title{
Прогнозирование физико-химических \\ и пожароопасных показателей \\ с помощью правил углеродной цепи.
}

\section{1. Алканали}

\author{
К.С. Алексеев ${ }^{\mathrm{a}, \boldsymbol{\sigma}}$, \\ С.Г. Алексеев ${ }^{\mathrm{a}, \mathrm{s}}$, Н.М. Барбин ${ }^{\text {б,в }}$ \\ ${ }^{a}$ НИЦ «Надежность и ресурс больших систем и машин» УрО РАН \\ Россия, 620049, Екатеринбург, ул. Студенческая, 54а

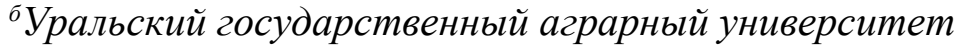 \\ Россия, 620075, Екатеринбург, ул. Карла Либкнехта, 42 \\ ${ }^{\circ}$ Уральский институт ГПС МЧС России \\ Россия, 620062, Екатеринбург, ул. Мира, 22
}

Проведено исследование зависимости физико-химических и пожароопасных свойств от химического строения алканалей. С помощьью правил углеродной цепи выполнено прогнозирование температур кипения и вспышки, температурных и концентрациионых пределов воспламенения, теплот сгорания и парообразования. Для удобства практического применения правил углеродной цепи предложены следующңе эмпирические уравнения для расчета физико-химических и пожароопасных показателей от условной углеродной цепи и от числа атомов углерода. Проведен сравнительный анализ предлагаемых методов расчета температуры вспышки с методами ГОСТ 12.1.044-89, Менделеева и ACD/Lab 2014. Показано, что новые методы в основном дают более точные результаты расчета, чем методы сравнения.

Ключевые слова: температура кипения, температура вспьики, температурные предель воспламенения, концеентрационные предель воспламенения, теплота сгорания, теплота парообразования, альдегид.

\section{Введение}

Показатели пожаровзрывоопасности играют важную роль в обеспечении промышленной безопасности. Бурное развитие химии, химической и нефтехимической промышленности приводит к открытию и использованию новых веществ и материалов. По данным Chemical Abstracts Service, на 26 февраля 2018 г. зарегистрировано более 137 млн индивидуальных соединений и более 67 млн субстанций сложного состава. Ежедневно регистрируется около 15 тыс. новых соединений [1]. В то же время физико-химические свойства в полной мере описаны для нескольких сотен тысяч соединений, а показатели пожарной опасности определены только для нескольких тысяч органических соединений. В связи с этим на расчетные методы возлагается задача восполнить существующий пробел в базе данных по показателям физико-химических свойств и пожаровзрывоопасности. 
Ранее нами отмечалось [2], что известные методы прогнозирования показателей физикохимических и пожарных свойств можно разбить на три основных типа - дескрипторный, сравнительный и правила углеродной цепи. Дескрипторный метод заключается в том, что какое-нибудь свойство соединения может быть выражено через функцию от одного или нескольких дескрипторов. В качестве последних могут выступать физико-химические, пожароопасные, структурные и молекулярные характеристики химических соединений [2-17]. Сравнительный метод, или Additivity Rules, разработан в середине XX столетия для теоретического определения различных физико-химических свойств органических соединений. Этот метод основан на использовании общего уравнения (1) или соотношения (2) [18-21].

$$
\begin{aligned}
& f_{2}=a \times f_{1}+b, \\
& \left\{\begin{array}{cc}
R_{1}-\Phi_{1} & R_{2}-\Phi_{1} \\
R_{1}-\Phi_{2} & R_{2}-\Phi_{2} \\
\Delta_{R_{1}} \approx \Delta_{R_{2}}
\end{array}\right\},
\end{aligned}
$$

где $f_{i}$ - физико-химический параметр $i$-класса химических соединений; $a, b$ - константы; $R_{i}-i$-радикал органической молекулы; $\Phi_{i}-i$-функциональная группа; $\Delta_{R_{i}}-$ количественная разница в значениях некого свойства между членами ряда.

Правила углеродной цепи (ПУЦ) представляют собой синтез-методику. В качестве предвестников этих правил можно считать гипотезы о подобии физико-химических дескрипторов и существовании линейных зависимостей между молекулярными дескрипторами родственных соединений одного гомологического ряда $[2,22,23]$. Из сравнительного метода прогнозирования ПУЦ заимствовали подход сравнения физико-химических и пожароопасных свойств в гомологическом ряду. Отличие в данном случае заключается в том, что сравнение производится не между родственными классами органических соединений, а только в пределах одного гомологического ряда. При этом с учетом уравнения (1) и соотношения (2) введено допущение, что свойства в одном классе соединений в пределах 2-3 ближайших гомологов нормального строения изменяются по линейному закону. Этот прием позволил ввести новые дескрипторы - основная углеродная цепь (ОУЦ) и условная углеродная цепь (УУЦ). Под ОУЦ понимается линейная углеводородная часть молекулы. УУЦ - это приведенная углеводородная цепь молекулы с учетом боковых алкильных заместителей. Для нормальных (линейных) соединений ОУЦ = УУЦ = $\mathrm{N}_{\mathrm{C}}$ (число атомов углерода в молекуле). В органической химии хорошо известно, что физико-химические характеристики изосоединений отличаются от их изомеров нормального строения. Выдвинута гипотеза, что $\mathrm{CH}_{3}$-группа в боковой углеродной цепи молекулы удлиняет ОУЦ не на 1 , а на 0,5 . В то время как другие атомы углерода увеличивают ОУЦ на 1. Следствие введенных допущений - свойства изомерных соединений можно предсказать по их линейным гомологам путем ввода поправочных коэффициентов непосредственно для длины углеродной цепи в молекуле и перемещение по углеродной цепи молекулы алкильного заместителя или функциональной группы не приводит к существенным изменениям свойств химического соединения. Необходимо отметить, что изомеризация органических молекул практически не оказывает влияние на такие показатели, как концентрационные пределы воспламенения и теплота сгорания. Различают два варианта ПУЦ - ручной вариант, или ПУЦ 1, когда свойства веществ определяются как

$$
-221-
$$


арифметически среднее между значениями показателей ближайших соседей по гомологическому ряду, и ПУЦ 2, в котором предусматривается использование уравнений на основе дескриптора УУЦ. Порядок применения методики ПУЦ 1 и ПУЦ 2 с примерами приведен в работах $[2,22,23]$.

\section{Объекты, методы и результаты исследования}

В качестве объектов исследования выбраны 22 алифатических альдегида (табл. 1). Данные по температуре кипения $\left(t_{\text {киn }}\right)$, температуре вспышки $\left(t_{\text {всn }}\right)$, температурным

Таблица 1. Справочные и расчетные данные физико-химических и пожароопасных свойств альдегидов

Table 1. Reference and calculated data of physicochemical and fire hazard properties of aldehydes

\begin{tabular}{|c|c|c|c|c|c|c|c|c|}
\hline \multirow{2}{*}{$\begin{array}{c}\text { Соединение, } \\
\text { номер (УУЦ) }\end{array}$} & $t_{\text {кип }}$ & $t_{\mathrm{Bc \Pi}}$ & $t_{\mathrm{H}}$ & $t_{\mathrm{B}}$ & $C_{u}$ & $C_{6}$ & $-1 \times H_{c 2}$ & $H_{\text {nap }}$ \\
\hline & \multicolumn{4}{|c|}{${ }^{\circ} \mathrm{C}$} & \multicolumn{2}{|c|}{$\%$ (об.) } & \multicolumn{2}{|c|}{ кДж/моль } \\
\hline 1 & 2 & 3 & 5 & 6 & 7 & 8 & 9 & 10 \\
\hline $\begin{array}{l}\text { Формальдегид } \\
\mathbf{1}(0,75)\end{array}$ & $\begin{array}{r}-19.3^{1} \\
-14.0^{2} \\
-15.7^{3} \\
-19.5^{4}\end{array}$ & $\begin{array}{l}-53.4^{1} \\
-55.1^{2} \\
-58.4^{3} \\
-90.2^{5} \\
-72.0^{6}\end{array}$ & $\begin{array}{l}-53.2^{1} \\
-57.5^{2} \\
-57.2^{3} \\
-69.5^{7}\end{array}$ & $\begin{array}{l}-26.2^{1} \\
-24.2^{2} \\
-28.7^{2} \\
-48.4^{8}\end{array}$ & $\begin{array}{l}7.0^{1} \\
7.2^{2} \\
7.3^{3} \\
8.3^{9}\end{array}$ & $\begin{array}{c}73.0^{1} \\
57.3^{1} \\
71.7^{2} \\
38.2^{10}\end{array}$ & $\begin{array}{l}526.8^{1} \\
525,2^{2} \\
500.0^{3} \\
486.8^{11}\end{array}$ & $\begin{array}{l}23.3^{1} \\
23.3^{2} \\
23.6^{3} \\
22.3^{12}\end{array}$ \\
\hline $\begin{array}{l}\text { Ацетальдегид } \\
2 \text { (2) }\end{array}$ & $\begin{array}{l}21.0^{1} \\
23.2^{2} \\
21.1^{3} \\
18.6^{4}\end{array}$ & $\begin{array}{l}-41.2^{1} \\
-46.3^{2} \\
-39.9^{3} \\
-57.4^{5} \\
-45.3^{6}\end{array}$ & $\begin{array}{l}-42.2^{1} \\
-46.3^{2} \\
-38.9^{3} \\
-44.1^{7}\end{array}$ & $\begin{array}{c}-7.1^{1} \\
-7.1^{2} \\
-8.3^{3} \\
-19.3^{8}\end{array}$ & $\begin{array}{l}4.0^{1} \\
4.8^{2} \\
3.7^{3} \\
4.0^{9}\end{array}$ & $\begin{array}{l}30.0^{1} \\
35.9^{2} \\
26.7^{3} \\
21.9^{10}\end{array}$ & $\begin{array}{l}1104.6^{1} \\
1067.0^{2} \\
1100.0^{3} \\
1147.9^{11}\end{array}$ & $\begin{array}{l}25.7^{1} \\
26.5^{2} \\
26.7^{3} \\
25.8^{12}\end{array}$ \\
\hline $\begin{array}{l}\text { Пропаналь } \\
\mathbf{3} \text { (3) }\end{array}$ & $\begin{array}{l}49.0^{1} \\
47.9^{2} \\
49.2^{3} \\
49.3^{4}\end{array}$ & $\begin{array}{l}-30.0^{1} \\
-26.1^{2} \\
-25.1^{3} \\
-34.6^{5} \\
-26.6^{6}\end{array}$ & $\begin{array}{l}-30.0^{1} \\
-28.0^{2} \\
-24.2^{3} \\
-26.7^{7}\end{array}$ & $\begin{array}{l}6.9^{1} \\
6.9^{2} \\
8.1^{3} \\
1.0^{8}\end{array}$ & $\begin{array}{l}2.6^{1} \\
3.0^{2} \\
2.5^{3} \\
2.7^{9}\end{array}$ & $\begin{array}{c}17.0^{1} \\
21.3^{2} \\
17.7^{3} \\
15.4^{10}\end{array}$ & $\begin{array}{l}1684.0^{1} \\
1702.8^{2} \\
1700.0^{3} \\
1809.0^{11}\end{array}$ & $\begin{array}{l}29.7^{2} \\
29.7^{2} \\
29.6^{3} \\
28.3^{12}\end{array}$ \\
\hline $\begin{array}{l}\text { Бутаналь } \\
\mathbf{4} \text { (4) }\end{array}$ & $\begin{array}{l}74.8^{1} \\
75.5^{2} \\
76.1^{3} \\
77.6^{4}\end{array}$ & $\begin{array}{c}-11.0^{1} \\
-9.0^{2} \\
-10.3^{3} \\
-13.6^{5} \\
-9.4^{6}\end{array}$ & $\begin{array}{c}-13.7^{1} \\
-9.0^{2} \\
-9.5^{3} \\
-10.7^{7}\end{array}$ & $\begin{array}{c}20.9^{1} \\
23.9^{2} \\
24.5^{3} \\
19.6^{8}\end{array}$ & $\begin{array}{l}1.9^{1} \\
2.1^{2} \\
1.9^{3} \\
2.0^{9}\end{array}$ & $\begin{array}{l}12.5^{1} \\
13.6^{2} \\
13.3^{3} \\
11.8^{10}\end{array}$ & $\begin{array}{l}2301.0^{1} \\
2297.0^{2} \\
2300.0^{3} \\
2470.0^{11}\end{array}$ & $\begin{array}{l}33.7^{1} \\
31.9^{2} \\
32.3^{3} \\
31.9^{12}\end{array}$ \\
\hline $\begin{array}{l}\text { Пентаналь } \\
\mathbf{5} \text { (5) }\end{array}$ & $\begin{array}{l}102.0^{1} \\
101.4^{2} \\
101.9^{3} \\
103.7^{4}\end{array}$ & $\begin{array}{c}12.0^{1,13,14} \\
8.0^{2} \\
4.6^{3} \\
8.5^{5} \\
8.7^{6}\end{array}$ & $\begin{array}{l}12^{1,4} \\
6.6^{2} \\
5.2^{3} \\
6.2^{7}\end{array}$ & $\begin{array}{l}40.9^{1} \\
39.9^{2} \\
40.8^{3} \\
39.2^{8}\end{array}$ & $\begin{array}{l}1.5^{1} \\
1.6^{2} \\
1.5^{3} \\
1.6^{9}\end{array}$ & $\begin{array}{l}10.1^{1} \\
10.6^{2} \\
10.6^{3} \\
9.6^{10}\end{array}$ & $\begin{array}{l}2910.0^{1} \\
2912.5^{2} \\
2900.0^{3} \\
3131.1^{11}\end{array}$ & $\begin{array}{c}34.0^{1} \\
35.1^{2} \\
34.9^{3} \\
34.3^{12}\end{array}$ \\
\hline $\begin{array}{l}\text { Гексаналь } \\
6(6)\end{array}$ & $\begin{array}{l}128.0^{1} \\
127.5^{2} \\
126.4^{3} \\
127.9^{4}\end{array}$ & $\begin{array}{l}26.9^{1} \\
23.5^{2} \\
19.4^{3} \\
29.6^{5} \\
26.0^{6} \\
\end{array}$ & $\begin{array}{l}26.9^{1} \\
23.5^{2} \\
19.9^{3} \\
22.3^{7}\end{array}$ & $\begin{array}{l}58.9^{1} \\
58.4^{2} \\
57.2^{3} \\
58.0^{8}\end{array}$ & $\begin{array}{l}1.3^{1} \\
1.3^{2} \\
1.3^{3} \\
1.3^{9}\end{array}$ & $\begin{array}{l}8.7^{1} \\
8.9^{2} \\
8.8^{3} \\
7.6^{10}\end{array}$ & $\begin{array}{l}3524.0^{1} \\
3523.0^{2} \\
3500.0^{3} \\
3792.2^{11}\end{array}$ & $\begin{array}{c}36.5^{1} \\
36.9^{2} \\
37.3^{3} \\
36.6^{12}\end{array}$ \\
\hline $\begin{array}{l}\text { Гептаналь } \\
7 \text { (7) }\end{array}$ & $\begin{array}{c}153.0^{1} \\
150.0^{2} \\
149.8^{3} \\
150.4^{4}\end{array}$ & $\begin{array}{l}35.0^{1} \\
39.0^{2} \\
34.2^{3} \\
49.9^{5} \\
42.6^{6}\end{array}$ & $\begin{array}{l}35.0^{1} \\
39.0^{2} \\
34.6^{3} \\
37.7^{7}\end{array}$ & $\begin{array}{l}75.8^{1} \\
75.4^{2} \\
73.5^{3} \\
76.1^{8}\end{array}$ & $\begin{array}{l}1.1^{1} \\
1.2^{2} \\
1.1^{3} \\
1.1^{9}\end{array}$ & $\begin{array}{l}7.7^{1} \\
7.8^{2} \\
7.5^{3} \\
7.0^{10}\end{array}$ & $\begin{array}{l}4136.0^{1} \\
4132.0^{2} \\
4100.0^{3} \\
4453.2^{11}\end{array}$ & $\begin{array}{l}39.7^{1} \\
39.1^{2} \\
39.5^{3} \\
38.7^{12}\end{array}$ \\
\hline
\end{tabular}


Продолжение табл. 1

Continuation Table 1

\begin{tabular}{|c|c|c|c|c|c|c|c|c|}
\hline 1 & 2 & 3 & 5 & 6 & 7 & 8 & 9 & 10 \\
\hline $\begin{array}{l}\text { Октаналь } \\
\mathbf{8}(8)\end{array}$ & $\begin{array}{l}172.0^{1} \\
172.7^{2} \\
172.1^{3} \\
163.4^{4}\end{array}$ & $\begin{array}{l}51.0^{1} \\
49.0^{2} \\
49.0^{3} \\
65.4^{5} \\
55.3^{6}\end{array}$ & $\begin{array}{l}51.0^{1} \\
49.0^{2} \\
49.3^{3} \\
49.3^{7}\end{array}$ & $\begin{array}{l}91.9^{1} \\
91.9^{2} \\
89.9^{3} \\
89.8^{8}\end{array}$ & $\begin{array}{l}1.0^{1} \\
1.0^{2} \\
1.0^{3} \\
1.0^{9}\end{array}$ & $\begin{array}{l}6.9^{1} \\
7.0^{2} \\
6.6^{3} \\
6.5^{10}\end{array}$ & $\begin{array}{l}4740.0^{1} \\
4743.0^{2} \\
4700.0^{3} \\
5114.3^{11}\end{array}$ & $\begin{array}{c}41.7^{1} \\
42.1^{2} \\
41.6^{3} \\
40.0^{12}\end{array}$ \\
\hline $\begin{array}{l}\text { Нонаналь } \\
9 \text { (9) }\end{array}$ & $\begin{array}{l}192.4^{1} \\
190.3^{2} \\
193.1^{3} \\
190.8^{4}\end{array}$ & $\begin{array}{l}63.0^{1} \\
68.0^{2} \\
63.8^{3} \\
82.0^{5} \\
69.0^{6}\end{array}$ & $\begin{array}{l}63.0^{1} \\
68.0^{2} \\
64.0^{3} \\
61.7^{7}\end{array}$ & $\begin{array}{l}107.9^{1} \\
108.4^{2} \\
106.3^{3} \\
104.5^{8}\end{array}$ & $\begin{array}{l}0.9^{1} \\
0.9^{2} \\
0.9^{3} \\
0.9^{9}\end{array}$ & $\begin{array}{l}6.3^{1} \\
6.4^{2} \\
5.9^{3} \\
6.0^{10}\end{array}$ & $\begin{array}{l}5350.0^{1} \\
5349.0^{2} \\
5300.0^{3} \\
5775.4^{11}\end{array}$ & $\begin{array}{l}44.5^{1} \\
43.5^{2} \\
43.5^{3} \\
42.7^{12}\end{array}$ \\
\hline $\begin{array}{l}\text { Деканаль } \\
\mathbf{1 0}(10)\end{array}$ & $\begin{array}{l}208.5^{1} \\
212.7^{2} \\
213.0^{3} \\
209.0^{4}\end{array}$ & $\begin{array}{l}85.0^{1} \\
79.5^{2} \\
78.6^{3} \\
95.1^{5} \\
79.8^{6}\end{array}$ & $\begin{array}{l}84.9^{1} \\
79.5^{2} \\
78.7^{3} \\
71.3^{7}\end{array}$ & $\begin{array}{l}124.9^{1} \\
124.4^{2} \\
122.6^{3} \\
116.2^{8}\end{array}$ & $\begin{array}{l}0.8^{1} \\
0.8^{2} \\
0.8^{3} \\
0.8^{9}\end{array}$ & $\begin{array}{l}5.8^{1} \\
5.9^{2} \\
5.3^{3} \\
5.7^{10}\end{array}$ & $\begin{array}{l}5958.0^{1} \\
5939.5^{2} \\
5900.0^{3} \\
6436.4^{11}\end{array}$ & $\begin{array}{l}45.2^{1} \\
45.4^{2} \\
45.2^{3} \\
44.5^{12}\end{array}$ \\
\hline $\begin{array}{l}\text { Ундеканаль } \\
11 \text { (11) }\end{array}$ & $\begin{array}{l}233.0^{1} \\
228.7^{2} \\
231.7^{3} \\
226.1^{4}\end{array}$ & $\begin{array}{c}96.0^{1} \\
93.0^{2} \\
93.4^{3} \\
115.0^{5} \\
96.1^{6}\end{array}$ & $\begin{array}{l}96.0^{1} \\
93.0^{2} \\
93.3^{3} \\
86.4^{7}\end{array}$ & $\begin{array}{l}140.9^{1} \\
139.4^{2} \\
139.0^{3} \\
133.8^{8}\end{array}$ & $\begin{array}{l}0.7^{1} \\
0.8^{2} \\
0.7^{3} \\
0.7^{9}\end{array}$ & $\begin{array}{l}5.4^{1} \\
5.5^{2} \\
4.8^{3} \\
5.3^{10}\end{array}$ & $\begin{array}{l}6529.0^{1} \\
6553.0^{2} \\
6500.0^{3} \\
7097.5^{11}\end{array}$ & $\begin{array}{l}46.3^{1} \\
46.6^{2} \\
46.7^{3} \\
46.3^{12}\end{array}$ \\
\hline $\begin{array}{l}\text { Додеканаль } \\
\mathbf{1 2}(12)\end{array}$ & $\begin{array}{l}248.9^{1} \\
250.0^{2} \\
249.2^{3} \\
242.2^{4}\end{array}$ & $\begin{array}{l}101.0^{1} \\
107.5^{2} \\
108.2^{3} \\
127.9^{5} \\
106.7^{6}\end{array}$ & $\begin{array}{c}101.0^{1} \\
107.5^{2} \\
108.0^{3} \\
95.9^{7}\end{array}$ & $\begin{array}{l}153.9^{1} \\
153.9^{2} \\
155.4^{3} \\
145.3^{8}\end{array}$ & $\begin{array}{l}0.7^{1} \\
0.7^{2} \\
0.6^{3} \\
0.7^{9}\end{array}$ & $\begin{array}{l}5.1^{1} \\
5.1^{2} \\
4.4^{3} \\
5.0^{10}\end{array}$ & $\begin{array}{c}7148.0^{1} \\
7154.5^{2} \\
7100.0^{3} \\
7758.6^{11}\end{array}$ & $\begin{array}{l}47.9^{1} \\
47.9^{2} \\
48.1^{3} \\
47.9^{12}\end{array}$ \\
\hline $\begin{array}{l}\text { Тридеканаль } \\
\mathbf{1 3} \text { (13) }\end{array}$ & $\begin{array}{l}267.0^{1} \\
264.8^{2} \\
265.6^{3} \\
257.3^{4}\end{array}$ & $\begin{array}{l}118.9^{1} \\
106.0^{2} \\
123.0^{3} \\
142.6^{5} \\
118.8^{6}\end{array}$ & $\begin{array}{l}118.9^{1} \\
106.0^{2} \\
122.7^{3} \\
106.9^{7}\end{array}$ & $\begin{array}{l}166.9^{1} \\
166.9^{2} \\
171.7^{3} \\
158.4^{8}\end{array}$ & $\begin{array}{l}0.6^{1} \\
0.7^{2} \\
0.6^{3} \\
0.6^{9}\end{array}$ & $\begin{array}{l}4.8^{1} \\
4.8^{2} \\
4.0^{3} \\
4.7^{10}\end{array}$ & $\begin{array}{l}7780.0^{1} \\
7767.0^{2} \\
7700.0^{3} \\
8419.6^{11}\end{array}$ & $\begin{array}{l}49.5^{1} \\
49.3^{2} \\
49.3^{3} \\
49.5^{12}\end{array}$ \\
\hline $\begin{array}{l}\text { Изобутаналь } \\
14 \text { (3.5) }\end{array}$ & $\begin{array}{l}64.1^{1} \\
61.9^{2} \\
62.8^{3} \\
67.1^{4}\end{array}$ & $\begin{array}{l}-19.0^{1} \\
-20.5^{2} \\
-17.7^{3} \\
-22.3^{5} \\
-16.5^{6}\end{array}$ & $\begin{array}{l}-19.5^{1} \\
-21.9^{2} \\
-16.8^{3} \\
-17.7^{7}\end{array}$ & $\begin{array}{l}11.9^{1} \\
13.9^{2} \\
16.3^{3} \\
11.9^{8}\end{array}$ & $\begin{array}{l}1.6^{1} \\
1.9^{2} \\
1.9^{3} \\
2.0^{9}\end{array}$ & $\begin{array}{l}11.0^{1} \\
12.5^{2} \\
13.3^{3} \\
11.8^{10}\end{array}$ & $\begin{array}{l}2291.3^{1} \\
2301.0^{2} \\
2300.0^{3} \\
2470.0^{11}\end{array}$ & $\begin{array}{c}30.8^{2} \\
31.0^{3} \\
30.9^{12}\end{array}$ \\
\hline $\begin{array}{l}\text { Изопентаналь } \\
\mathbf{1 5}(4.5)\end{array}$ & $\begin{array}{l}92.5^{1} \\
88.4^{2} \\
89.1^{3} \\
93.5^{4}\end{array}$ & $\begin{array}{c}-3.0^{1} \\
0.5^{2} \\
-2.8^{3} \\
0.7^{5} \\
2.4^{6}\end{array}$ & $\begin{array}{c}-3.2^{1} \\
-0.9^{2} \\
-2.1^{3} \\
0.0^{7}\end{array}$ & $\begin{array}{l}25.6^{1} \\
30.9^{2} \\
32.6^{3} \\
32.4^{8}\end{array}$ & $\begin{array}{l}1.2^{1} \\
1.5^{2} \\
1.5^{3} \\
1.6^{9}\end{array}$ & $\begin{array}{c}12.9^{1} \\
10.1^{2} \\
10.6^{3} \\
9.6^{10}\end{array}$ & $\begin{array}{c}5348.0^{1,13} \\
2910.0^{2} \\
2900.0^{3} \\
3131.1^{11}\end{array}$ & $\begin{array}{c}33.5^{2} \\
33.6^{3} \\
33.3^{12}\end{array}$ \\
\hline $\begin{array}{l}\text { 2-Метилбутаналь } \\
\mathbf{1 6}(4.5)\end{array}$ & $\begin{array}{l}91.7^{1} \\
88.4^{2} \\
89.1^{3} \\
93.5^{4}\end{array}$ & $\begin{array}{c}-4.0^{1} \\
0.5^{2} \\
-2.8^{3} \\
0.1^{5} \\
1.9^{6}\end{array}$ & $\begin{array}{l}-6.2^{1} \\
-0.9^{2} \\
-2.1^{3} \\
-0.6^{7}\end{array}$ & $\begin{array}{l}36.9^{1} \\
30.9^{2} \\
32.6^{3} \\
31.8^{8}\end{array}$ & $\begin{array}{l}1.2^{1} \\
1.5^{2} \\
1.5^{3} \\
1.6^{9}\end{array}$ & $\begin{array}{l}12.9^{1} \\
10.1^{2} \\
10.6^{3} \\
9.6^{10}\end{array}$ & $\begin{array}{l}2905.0^{1} \\
2910.0^{2} \\
2900.0^{3} \\
3131.1^{11}\end{array}$ & $\begin{array}{c}33.5^{2} \\
33.6^{3} \\
33.3^{12}\end{array}$ \\
\hline $\begin{array}{l}\text { 2-Метилгексаналь } \\
17 \text { (6.5) }\end{array}$ & $\begin{array}{l}142.0^{1} \\
140.5^{2} \\
138.3^{3} \\
141.2^{4}\end{array}$ & $\begin{array}{l}34.9^{1} \\
31.0^{2} \\
26.8^{3} \\
41.0^{5} \\
35.4^{6}\end{array}$ & $\begin{array}{l}32.9^{1} \\
31.0^{2} \\
27.2^{3} \\
30.5^{7}\end{array}$ & $\begin{array}{l}68.9^{1} \\
67.3^{2} \\
65.4^{3} \\
68.1^{8}\end{array}$ & $\begin{array}{l}1.1^{1} \\
1.1^{2} \\
1.1^{3} \\
1.1^{9}\end{array}$ & $\begin{array}{l}7.2^{1} \\
7.7^{2} \\
7.5^{3} \\
7.0^{10}\end{array}$ & $\begin{array}{l}4130.0^{1} \\
4136.0^{2} \\
4100.0^{3} \\
4453.2^{11}\end{array}$ & $\begin{array}{l}38.0^{2} \\
38.4^{3} \\
37.8^{12}\end{array}$ \\
\hline
\end{tabular}


Продолжение табл. 1

Continuation Table 1

\begin{tabular}{|c|c|c|c|c|c|c|c|c|}
\hline 1 & 2 & 3 & 5 & 6 & 7 & 8 & 9 & 10 \\
\hline $\begin{array}{l}\text { 3-Метилгексаналь } \\
\mathbf{1 8}(6.5)\end{array}$ & $\begin{array}{l}144.3^{1} \\
140.5^{2} \\
138.3^{3} \\
141.2^{4}\end{array}$ & $\begin{array}{l}29.2^{1} \\
31.0^{2} \\
26.8^{3} \\
42.9^{5} \\
36.9^{6}\end{array}$ & $\begin{array}{l}27.9^{1} \\
31.0^{2} \\
27.2^{3} \\
32.0^{7}\end{array}$ & $\begin{array}{l}57.9^{1} \\
67.3^{2} \\
65.4^{3} \\
69.8^{8}\end{array}$ & $\begin{array}{l}0.9^{1} \\
1.1^{2} \\
1.1^{3} \\
1.1^{9}\end{array}$ & $\begin{array}{l}6.6^{1} \\
7.7^{2} \\
7.5^{3} \\
7.0^{10}\end{array}$ & $\begin{array}{l}4130.0^{1} \\
4136.0^{2} \\
4100.0^{3} \\
4453.2^{11}\end{array}$ & $\begin{array}{c}38.0^{2} \\
38.4^{3} \\
37.8^{12}\end{array}$ \\
\hline $\begin{array}{l}\text { 2-Этилгексаналь } \\
\mathbf{1 9}(7.5)\end{array}$ & $\begin{array}{l}163.0^{1} \\
162.5^{2} \\
161.1^{3} \\
163.0^{4}\end{array}$ & $\begin{array}{l}44.4^{1} \\
43.0^{2} \\
41.6^{3} \\
58.1^{5} \\
49.4^{6}\end{array}$ & $\begin{array}{l}43.7^{1} \\
43.0^{2} \\
41.9^{3} \\
43.4^{7}\end{array}$ & $\begin{array}{l}83.5^{1} \\
83.9^{2} \\
81.7^{3} \\
83.3^{8}\end{array}$ & $\begin{array}{l}0.9^{1} \\
1.0^{2} \\
1.0^{3} \\
1.0^{9}\end{array}$ & $\begin{array}{l}6.6^{1} \\
6.9^{2} \\
6.6^{3} \\
6.5^{10}\end{array}$ & $\begin{array}{l}4734.4^{1} \\
4740.0^{2} \\
4700.0^{3} \\
5114.3^{11}\end{array}$ & $\begin{array}{l}40.6^{2} \\
40.6^{3} \\
40.0^{12}\end{array}$ \\
\hline $\begin{array}{l}\text { 2-Метилгептаналь } \\
\mathbf{2 0}(7.5)\end{array}$ & $\begin{array}{l}156.0^{1} \\
162.5^{2} \\
161.1^{3} \\
163.0^{4}\end{array}$ & $\begin{array}{l}46.9^{1} \\
43.0^{2} \\
41.6^{3} \\
52.4^{5} \\
44.8^{6}\end{array}$ & $\begin{array}{l}44.9^{1} \\
43.0^{2} \\
41.9^{3} \\
38.8^{7}\end{array}$ & $\begin{array}{l}87.9^{1} \\
83.9^{2} \\
81.7^{3} \\
78.2^{8}\end{array}$ & $\begin{array}{l}0.9^{1} \\
1.0^{2} \\
1.0^{3} \\
1.0^{9}\end{array}$ & $\begin{array}{l}8.3^{1} \\
6.9^{2} \\
6.6^{3} \\
6.5^{10}\end{array}$ & $\begin{array}{l}4703.0^{1} \\
4740.0^{2} \\
4700.0^{3} \\
5114.3^{11}\end{array}$ & $\begin{array}{l}40.6^{2} \\
40.6^{3} \\
40.0^{12}\end{array}$ \\
\hline $\begin{array}{l}\text { 2-Метилоктаналь } \\
21(8.5)\end{array}$ & $\begin{array}{l}186.9^{1} \\
182.2^{2} \\
182.7^{3} \\
183.7^{4}\end{array}$ & $\begin{array}{l}57.9^{1} \\
57.0^{2} \\
56.4^{3} \\
77.5^{5} \\
65.3^{6}\end{array}$ & $\begin{array}{l}55.9^{1} \\
57.0^{2} \\
56.6^{3} \\
58.1^{7}\end{array}$ & $\begin{array}{c}111.5^{1} \\
99.9^{2} \\
98.1^{3} \\
100.6^{8}\end{array}$ & $\begin{array}{l}1.1^{1} \\
0.9^{2} \\
0.9^{3} \\
0.9^{9}\end{array}$ & $\begin{array}{l}7.2^{1} \\
6.3^{2} \\
5.9^{3} \\
6.0^{10}\end{array}$ & $\begin{array}{l}5348.0^{1} \\
5350.0^{2} \\
5300.0^{3} \\
5775.4^{11}\end{array}$ & $\begin{array}{l}42.8^{2} \\
42.5^{3} \\
42.0^{12}\end{array}$ \\
\hline $\begin{array}{l}\text { 3-Этилгептаналь } \\
22 \text { (8.5) }\end{array}$ & $\begin{array}{l}180.4^{1} \\
182.2^{2} \\
182.7^{3} \\
183.7^{4}\end{array}$ & $\begin{array}{l}57.9^{1} \\
57.0^{2} \\
56.4^{3} \\
72.2^{5} \\
61.1^{6}\end{array}$ & $\begin{array}{l}55.9^{1} \\
57.0^{2} \\
56.6^{3} \\
53.8^{7}\end{array}$ & $\begin{array}{c}103.9^{1} \\
99.9^{2} \\
98.1^{3} \\
95.9^{8}\end{array}$ & $\begin{array}{l}0.9^{1} \\
0.9^{2} \\
0.9^{3} \\
0.9^{9}\end{array}$ & $\begin{array}{l}6.6^{1} \\
6.3^{2} \\
5.9^{3} \\
6.0^{10}\end{array}$ & $\begin{array}{l}5348.0^{1} \\
5350.0^{2} \\
5300.0^{3} \\
5775.4^{11}\end{array}$ & $\begin{array}{l}42.8^{2} \\
42.5^{3} \\
42.0^{12}\end{array}$ \\
\hline
\end{tabular}

Примечания. 'Данные DIPPR 801. ${ }^{2-12}$ Расчетные значения по ПУЦ 1, ПУЦ 2 (уравнения 1-8), ACD/Lab $\left(t_{\text {киn }}\right)$, уравнения 9-13 и ACD/Lab $\left(H_{n a p}\right) .{ }^{13}$ Ошибочные данные DIPPR 801, которые не учитывались при расчетах. ${ }^{14}$ Данные ChemSpider [25] для соединения 5 дают целый набор значений температуры вспышки $\left\{4,8\right.$ и $\left.12{ }^{\circ} \mathrm{C}\right\}$.

пределам воспламенения $\left(t_{t}, t_{6}\right)$, концентрационным пределам воспламенения $\left(C_{t}\right.$, $\left.C_{6}\right)$, теплоте сгорания $\left(H_{c 2}\right)$ и теплоте парообразования $\left(H_{n a p}\right)$ взяты из базы данных DIPPR 801 [24].

В качестве метода исследования выбраны ПУЦ 1 и ПУЦ 2. Для вывода уравнений для ПУЦ 2 исследуемая группа соединений была разбита на обучающую и контрольную выборки. В первую были включены нормальные соединения (1)-(13), а во вторую - соединения изостроения. В результате математической обработки данных обучающей выборки с помощью программы M.Excel 2010 получены уравнения (1)-(8) для прогнозирования температуры кипения и вспышки, температурных и концентрационных пределов воспламенения, теплот сгорания и парообразования алканалей (табл. 2), по которым выполнен прогноз физико-химических и пожароопасных показателей для изоалканалей (14)-(22) (табл. 1).

Каждый класс органических соединений может иметь некоторые индивидуальные особенности, и класс алифатических альдегидов не является исключением. Для родоначальника этого класса (формальдегида (1)) введена поправка -0,25, поэтому его УУЦ равна не 1, а 0,75. Данная поправка также была учтена при расчете концентрационных пределов воспламенения для соединения $\mathbf{1}$ по ПУЦ 1, что позволило значительно улучшить коэф- 
Таблица 2. Методы ПУЦ 1, ПУЦ 2 и уравнения сравнения

Table 2. The methods of RCC 1, RCC 2 and the comparison equations

\begin{tabular}{|c|c|c|c|c|}
\hline Уравнение / метод & & $\mathrm{r}$ & SD & AAEP \\
\hline ПУЦ $1\left(t_{\text {кии }}\right)$ & - & 0.9991 & 3.08 & 3.36 \\
\hline$t_{\text {кun }}=-0.587(\text { УУЦ })^{2}+31.03($ УУЦ $)-38.6($ ПУЦ 2) & (1) & 0,9993 & 2.81 & 2.36 \\
\hline $\mathrm{ACD} / \mathrm{Lab}\left(t_{\text {кии }}\right)$ & - & 0.9988 & 4.16 & 2.46 \\
\hline ПУЦ $1\left(t_{\text {вcn }}\right)$ & - & 0.9955 & 4.50 & 18.04 \\
\hline$t_{\text {scn }}=14.8($ УУЦ) -69.45 (ПУЦ 2) & (2) & 0.9962 & 4.06 & 8.95 \\
\hline$t_{\text {вcn }}=a t_{\text {кип }}+b$ & (9) & 0.9933 & 15.84 & 36.97 \\
\hline$t_{\text {вcn }}=a_{0}+a_{l} \cdot t_{\text {кип }}+\sum a_{i} \cdot l_{i}$ & (10a) & 0.9934 & 6.08 & 25.14 \\
\hline ПУЦ $1\left(t_{H}\right)$ & - & 0.9952 & 4.49 & 15.23 \\
\hline$t_{H}=14.689 \times(У У Ц)-68.24$ (ПУЦ 2) & (3) & 0.9961 & 3.86 & 12.54 \\
\hline$t_{H}=a_{0}+a_{i} \cdot t_{\text {кun }}+\sum a_{i} \cdot l_{i}$ & $(106)$ & 0.9920 & 6.47 & 19.62 \\
\hline ПУЦ $1\left(t_{B}\right)$ & - & 0.9973 & 3.95 & 5.36 \\
\hline$t_{6}=16.363 \times($ УУЦ $)-41$ & (4) & 0.9961 & 4.67 & 8.99 \\
\hline$t_{B}=a_{0}+a_{1} \cdot t_{\text {кии }}+\sum a_{i} \cdot l_{i}$ & $(10 \mathrm{~B})$ & 0.9914 & 8.15 & 21.52 \\
\hline ПУЦ $1\left(C_{H}\right)$ & - & 0.9704 & 0.35 & 10.58 \\
\hline$C_{H}=7.327 \times N_{c}^{-0.98}$ & (5) & 0.9938 & 0.16 & 8.05 \\
\hline$C_{H}=100 / \sum_{s=1}^{q} h_{s} m_{s}$ & (11) & 0.9933 & 0.32 & 9.72 \\
\hline ПУЦ $1\left(C_{B}\right)$ & - & 0.9382 & 6.00 & 10.06 \\
\hline$C_{6}=53.67 \times N_{c}^{-1.008}$ & (6) & 0.9892 & 1.32 & 9.96 \\
\hline $\begin{array}{l}C_{6}=100\left(\sum_{j=1}^{l} h_{j} m_{j}+\sum_{s=1}^{q} q_{s}\right) \quad n p u \beta \leq 8 \\
C_{6}=100 /(0,768 \beta+6,554) \quad \text { при } \beta>8\end{array}$ & $\begin{array}{l}(12 \mathrm{a}) \\
(126)\end{array}$ & 0.9795 & 7.72 & 11.77 \\
\hline ПУЦ $1\left(H_{c 2}\right)$ & - & 1.0000 & 14.72 & 0.39 \\
\hline$H_{c z}=-600 \times N_{c}+100$ & (7) & 0.9977 & 36.30 & 0.85 \\
\hline$H_{c 2}=-(339.4 C+1257 H-108.9 O)$ в кДж/кг & $(13)$ & 1.000 & 371.28 & 7.74 \\
\hline ПУЦ $1\left(H_{\text {nap }}\right)$ & - & 0.9913 & 0.74 & 1.47 \\
\hline$H_{n a p}=-0.085(\text { УУЦ })^{2}+3.332(У У Ц)+20.36$ & (8) & 0.9925 & 0.67 & 1.48 \\
\hline $\mathrm{ACD} / \mathrm{Lab}\left(H_{\text {nap }}\right)$ & - & 0.9958 & 1.04 & 2.14 \\
\hline
\end{tabular}

Примечания. $t_{\text {кип }} t_{s c n}$ - температуры кипения и вспышки (закрытый тигель), ${ }^{\circ} \mathrm{C} ; t_{H} \cdot t_{B}-$ нижний и верхний температурные пределы воспламенения, ${ }^{\circ} \mathrm{C} ; C_{H} C_{B}-$ нижний и верхний концентрационные пределы воспламенения, \%; $H_{c 2}$ - высшая теплота (энтальпия) сгорания, кДж/моль; $N_{c}$ - число атомов углерода в молекуле вещества; $a, b, a_{0}, a_{1}$ - константы [26]; $h_{j} m_{j}, h_{s} m_{s}, a_{i} l_{i}$ - произведение вкладов атомных связей на их количество [26]; $\beta$ - стехиометрический коэффициент перед кислородом в реакции полного горения горючего вещества, $\beta=N_{c}+0.25 N_{H}-0.5 N_{O}[26]$.

фициент корреляции $r$. В качестве уравнения сравнения взяты формулы (9)-(12) из ГОСТ 12.1.044 [26] для прогнозирования температуры вспышки, температурных и концентрационных пределов воспламенения. Ранее было показано, что формула Менделеева (13) для определения теплоты сгорания дает приемлемые результаты расчета [27], поэтому она использована в качестве метода сравнения. Для сравнения прогнозов температуры кипения 
и теплоты парообразования алканалей (1)-(22) использован программный комплекс $A C D /$ Lab 2014 [27].

\section{Обсуждение результатов}

В результате критического анализа данных DIPPR 801 (табл. 1) выявлены некорректные значения температуры вспышки для пентаналя (5) и теплоты сгорания для изопентаналя (14). В связи с этим они не учитывались в настоящем исследовании. Сопоставление расчетных данных табл. 1 и 2 показывает, что коэффициент корреляции $(r)$ плохо подходит в качестве арбитра для выявления лучшего метода прогнозирования физико-химических и пожароопасных характеристик алканалей, поэтому в качестве критериев сравнения выбраны среднее отклонение $S D$ (Standard Deviation) и средняя процентная ошибка AAPE (Average Absolute Percent Error) [28].

$$
\begin{aligned}
& S D=\sqrt{\frac{\sum\left(X_{\text {pacч }}-X_{\text {экс }}\right)^{2}}{N}}, \\
& A A P E=\frac{1}{N} \sum \frac{\left|\left(X_{\text {расч }}-X_{\text {экс }}\right)\right|}{\left|X_{\text {экс }}\right|} \cdot 100 \% .
\end{aligned}
$$

Из данных табл. 2 видно, что методы ПУЦ 1 и ПУЦ 2 по показателям $S D$ и $A A P E$ дают лучший прогноз физико-химических и пожароопасных свойств алканалей по сравнению нормативными методами ГОСТ 12.1.044, уравнением Менделеева и программой ACD/Lab 2014. Исключение составляет только ПУЦ 1 для расчета температуры кипения и нижнего концентрационного предела воспламенения, который незначительно уступает методам сравнения.

В ГОСТ 12.1.044 [25] заложены стандартные экспериментальные ошибки при определении показателей пожарной опасности (табл. 3). Оценивая в целом методы ПУЦ 1 и ПУЦ 2 по прогнозированию показателей пожарной опасности, можно сделать вывод, что они в основном укладываются в данные экспериментальные допуски.

Таблица 3. Допустимые экспериментальные ошибки по ГОСТ 12.1.044-89

\begin{tabular}{|c|c|c|c|}
\hline Показатель & Сходимость ${ }^{1}$ & Воспроизводимость ${ }^{2}$ & $\begin{array}{c}\text { Область применения } \\
\text { метода }\end{array}$ \\
\hline$t_{\text {всп }}$ & $\begin{array}{l}2.0^{\circ} \mathrm{C}\left(\text { при } \mathrm{t}_{\text {всп }} \leq 104^{\circ} \mathrm{C}\right) \\
5.5^{\circ} \mathrm{C}\left(\text { при } \mathrm{t}_{\text {всп }}>104^{\circ} \mathrm{C}\right)\end{array}$ & $\begin{array}{l}3.5^{\circ} \mathrm{C}\left(\text { при } \mathrm{t}_{\text {всп }} \leq 104^{\circ} \mathrm{C}\right) \\
8.0^{\circ} \mathrm{C}\left(\text { при } \mathrm{t}_{\text {всп }}>104^{\circ} \mathrm{C}\right)\end{array}$ & $-15 \ldots 360^{\circ} \mathrm{C}$ \\
\hline$C_{u}$ & $0.1 \%$ & $0.3 \%$ & \multirow{2}{*}{$15 \ldots 150^{\circ} \mathrm{C}$} \\
\hline$C_{6}$ & $0.2 \%$ & $0.6 \%$ & \\
\hline$t_{u}$ & $7^{\circ} \mathrm{C}$ & $15^{\circ} \mathrm{C}$ & \multirow{2}{*}{$-15 \ldots 300^{\circ} \mathrm{C}$} \\
\hline$t_{6}$ & $7^{\circ} \mathrm{C}$ & $15^{\circ} \mathrm{C}$ & \\
\hline
\end{tabular}

Table 3. Reasonable experimental errors in accordance with GOST 12.1.044-89

Примечания. ${ }^{1}$ Сходимость - расхождение в результатах параллельных тестов, проводимых на одной установке и в одной лаборатории. ${ }^{2}$ Воспроизводимость - расхождение в результатах тестов, проводимых на разных установках и лабораториях. 


\section{Заключение}

В результате проведенного исследования найдено, что предлагаемые методы ПУЦ 1 и ПУЦ 2 могут быть использованы для расчета физико-химических и пожароопасных свойств алканалей. По точности прогнозов температуры вспышки вторичных аминов правила углеродной цепи превосходят методы сравнения (ГОСТ 12.1.044, уравнение Менделеева, ACD/Lab 2014), кроме ПУЦ 1, для расчета температуры кипения и нижнего концентрационного предела воспламенения, который практически сопоставим с методам сравнения ( $A C D / L a b 2014$ и ГОСТ 12.1.044).

\section{Список литературы}

1. CAS REGISTRY - The gold standard for chemical substance information. Available at http:// www.cas.org/content/chemical-substances.

2. Смирнов В.В., Алексеев С.Г., Барбин Н.М. Прогнозирование температуры вспышки диалкиламинов. Журнал Сибирского федерального университета. Химия. 2016. Т. 9(1), С. 68-77. [Smirnov V.V., Alexeev S.G., Barbin N.M. Prediction of the dialkylamine's flash points. Journal of the Siberian Federal University. Chemistry. 2016. Vol. 9(1), P. 68-77. (In Russ.)]

3. Katritzky A.R., Kuanar M., Slavov S., Hall C.D., Karelson M., Dobchev D. Quantitative correlation of physical and chemical properties with chemical structure: utility for prediction. Chem. Rev. 2010. Vol. 110(10), P. 5714-5789.

4. Nieto-Draghi C., Fayet G., Creton B., Rozanska X., Rotureau P., de Hemptinne J.-Ch., Ungerer P., Rousseau B., Adamo C. A general guidebook for the theoretical prediction of physicochemical properties of chemicals for regulatory purposes. Chem. Rev. 2015. Vol. 115(24), P. 13093-13164.

5. Su W., Zhao L., Deng S. Group contribution methods in thermodynamic cycles: Physical properties estimation of pure working fluids. Renewable \& Sustainable Energy Rev. 2017. Vol. 79, P. 984-1001.

6. Батов Д.В., Сторонкина О.Е., Мочалова Т.А. Модификация аддитивно-группового метода для описания показателей пожарной опасности предельных углеводородов: температур вспышки и самовоспламенения, энтальпии испарения. Пожаровзрывобезопасность. 2017. Т. 26(5), С. 21-28. [Batov D.V., Storonkina O.E., Mochalova T.A. Modification of the additive-group method to describe the fire hazard indicators of paraffin hydrocarbons: flash point and spontaneous ignition temperature, vaporation enthalpy. Pozharovzryvobezopasnost’. 2017. Vol. 26(5), P. 21-28. (In Russ.)]

7. Королев Д.С., Калач А.В. Прогнозирование пожароопасных свойств веществ. Воронеж: Воронежский институт ГПС МЧС России, 2018. 100 c. [Korolev D.S., KalachA.V. Prediction of fire properties of substances. Voronezh: Voronezh State Fire Service Institute of EMERCOM of Russia, 2018. 100 p. (In Russ.)]

8. Potemkin V., Grishina M. Electron-based descriptors in the study of physicochemical properties of compounds. Computational and Theoretical Chem. 2018. Vol. 1123, P. 1-10.

9. Yan F., He W., Jia Q., Wang Q., Xia S., Ma P. Prediction of ionic liquids viscosity at variable temperatures and pressures. Chem. Eng. Sci. 2018. Vol. 184, P. 134-140.

10. Ojha P.K., Roy K. Development of a robust and validated 2D-QSPR model for sweetness potency of diverse functional organic molecules. Food and Chemical Toxicology. 2018. Vol. 112, P. 551-562.

$$
-227-
$$


11. Rybinska-Fryca A., Sosnowska A., Puzyn T. Prediction of dielectric constant of ionic liquids. J. Mol. Liq. 2018. Vol. 260, P. 57-64.

12. Krasnykh E.L., Druzhinina Y.A., Portnova S.V., Smirnova Y.A. Vapor pressure and enthalpy of vaporization of trimethylolpropane and carboxylic acids esters. Fluid Phase Equilibria. 2018. Vol. 462, P. 111-117.

13. Keshavarz M.H., Jafari M., Esmaeilpour K., Samiee M. New and reliable model for prediction of autoignition temperature of organic compounds containing energetic groups. Process Safety and Environmental Protection. 2018. Vol. 113, P. 491-497.

14. Jhamb S., Liang X., Gani R., Hukkerikar A.S. Estimation of physical properties of amino acids by group-contribution method. Chem. Eng. Sci. 2018. Vol. 175, P. 148-161.

15. Alantary D., Yalkowsky S.H. Estimating the physicochemical properties of polysubstituted aromatic compounds using UPPER. J. Pharm. Sci. 2018. Vol. 107(1), P. 297-306.

16. Cao L., Zhu P., Zhao Y., Zhao J. Using machine learning and quantum chemistry descriptors to predict the toxicity of ionic liquids. J. Hazardous Mat. 2018. Vol. 352, P. 17-26.

17. Zhang X., Cheng D., Shi J., Qin L., Wang T., Fang B. QSPR modeling of the logKow and $\log K o c$ of polymethoxylated, polyhydroxylated diphenyl ethers and methoxylated-, hydroxylated-polychlorinated diphenyl ethers. J. Hazardous Mater. 2018. In press. DOI: 10.1016/j. jhazmat.2018.03.043.

18. Карапетьянц М.Х. Методы сравнительного расчета физико-химических свойств. М.: Наука, 1965. 404 с. [Karapet'yants M.Kh. Methods for the Relative Prediction Physicochemical Properties. Moscow: Nauka, 1965. 404 p. (In Russ.)]

19. Benson S.W., Cruickshank F.R., Golden D.M., Haugen G.R., O’Neal H.E., Rodgers A.S., Shaw R., Walsh R. Additivity rules for the estimation of thermochemical properties, Chem. Rev. 1969. Vol. 69(3), P. 279-324.

20. Kearsley S.K., Sallamack S., Fluder E.M., Andose J.D., Mosley R.T., Sheridan R.P. Chemical similarity using physiochemical property descriptors. J. Chem. Inf. Comput. Sci. 1996. Vol. 36(1), P. 118-127.

21. Shacham M., Brauner N., Cholakov G.St., Stateva R.P. Property prediction by correlations based on similarity of molecular structures. AIChE J. 2004. Vol. 50(10), P. 2481-2492.

22. Алексеев К.С. Применение правил углеродной цепи и дескрипторного метода для расчета показателей пожарной опасности кислородсодержащих органических соединений: дис. ... канд. техн. наук. Уфа, 2016, 155 c. [Alexeev K.S. The application of the rules of the carbon chain and the descriptor method for calculating the fire hazard indices of oxygen-containing organic compounds: PhD (technology) Diss. Ufa, 2016. 155 p. (In Russ.). Available at: http://new.rusoil.net/files/1006/ AlekseevKS/Дисс.Алексеев Уфа 01_07.pdf]

23. Alexeev K.S., Alexeev S.G., Barbin N.M. Correlation of fire hazard characteristics with chemical structure. Part XXII. Dialkyl carbonates. Butlerov Communications. 2016. Vol. 45. No. 1. P. 93-100.

24. DIPPR 801. Available at: https://dippr.aiche.org/.

25. Chem Spider. Available at: http://www.chemspider.com.

26. ГОСТ 12.1.044-89*. ССБТ. Пожаровзрывоопасность веществ и материалов. Номенклатура показателей и методы их определения. М.: Стандартинформ. 2006. 100 с. [State Standard 
12.1.044-89*. Occupational Safety Standards System. Fire and Explosion Hazard of Substances and Materials. Moscow: Standartinform, 2006. 100 p. (In Russ.)].

27. Демидов П.Г., Саушев В.С. Горение и свойства горючих веществ. М.: ВИПТШ МВД CCCP, 1975. 280 c. [Demidov P.G., Saushev V.S. Combustion and properties of combustible substances. Moscow: VIPTShMVD SSSR, 1975. 280 p. (In Russ.)]

28. ACD/Boiling Point. Version for Microsoft Windows. User's guide. Calculating the boiling point, vapor pressure, and related properties. Toronto: Advanced Chemistry Development, 2013. 29 p.

29. Stefanis E., Constantinou L., Panayiotou C. A group-contribution method for predicting pure component properties of biochemical and safety interest. Ind. Eng. Chem. Res. 2004. Vol. 43(19), P. 6253-6261. 